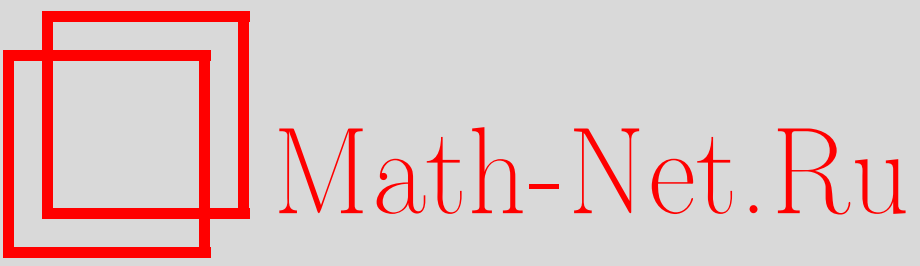

А. Ю. Голубков, Радикалы группы Шевалле над коммутативным кольцом, УМH, 2000, том 55, выпуск 3, 173-174

DOI: https://doi.org/10.4213/rm298

Использование Общероссийского математического портала Math-Net.Ru подразумевает, что вы прочитали и согласны с пользовательским соглашением

http://www.mathnet.ru/rus/agreement

Параметры загрузки:

IP: 54.198 .187 .58

26 апреля 2023 г., 18:10:30 


\title{
РАДИКАЛЫ ГРУППЫ ШЕВАЛЛЕ НАД КОММУТАТИВНЫМ КОЛЬЦОМ
}

\author{
А. Ю. ГолуБков
}

Введение. Основной целью настоящей работы является описание первичного радикала группы Шевалле над произвольным коммутативным кольцом с единицей (за исключением случая $A_{1}$, когда существенным является предположение об обратимости 2 и 3 ). В качестве следствия получено описание разрешимого радикала группы Шевалле. Отметим также, что близкие по характеру результаты о строении нормальных подгрупп группы Шевалле в терминах идеалов (см., например [1], [2]) требуют, как правило, более сильных ограничений на кольцо коэффициентов.

Первичный и разрешимый радикалы группы Шевалле. Всюду ниже $R$ - коммутативное кольцо с единицей и первичным радикалом $B(R) . L$-полупростая комплексная алгебра Ли с корневой системой $\Sigma$ и базисом Шевалле $\left\{X_{\alpha}, H_{i}\right\}_{\alpha \in \Sigma \backslash\{0\}, 1 \leqslant i \leqslant l}$, где $l=\operatorname{rk}(\Sigma)$ - мощность фундаментальной подсистемы в $\Sigma$.

ОпредЕлЕниЕ 1 . В неприводимом точном комплексном представлении $\pi$ алгебры $L$ определена целочисленная решетка базисных векторов $V$, инвариантная относительно действия операторов $\left\{\pi\left(X_{\alpha}^{n} / n !\right)\right\}$, представляющих соответствующие элементы ее универсальной обертывающей (см. [3; теорема Шевалле-Ри]). Группа $E(\pi, \Sigma, R)$, порожденная всеми автоморфизмами модуля $R \otimes_{\mathbb{Z}} V$ ( $\mathbb{Z}$ - кольцо целых чисел) вида

$$
x_{\alpha}(r)=\sum_{n=0}^{\infty} r^{n} \pi\left(X_{\alpha}^{n} / n !\right),
$$

где $r \in R, \alpha \in \Sigma \backslash\{0\}$, называется элементарной группой Шевалле, определенной представлением $\pi$, системой $\Sigma$ и кольцом $R$.

В частности, в присоединенном представлении алгебры $L$ участвующая в данном определении решетка $V$ задается самим базисом Шевалле.

Над полем комплексных чисел $\mathbb{C}$ элементарная группа Шевалле совпадает с алгебраической матричной группой над полем рациональных чисел $\mathbb{Q}$, представленной в базисе решетки $V$ (см. [3]-[5]). Соответствующий идеал $I$ в кольце целочисленных многочленов от $n^{2}$-переменных $(n=\operatorname{dim} \pi) \mathbb{Z}\left[x_{11}, \ldots, x_{i j}, \ldots, x_{n n}\right]$ состоит из всех полиномов, обрашающихся в нуль на всех элементах $E(\pi, \Sigma, \mathbb{C})$. Фактор-кольцо $\mathbb{Z}\left[x_{11}, \ldots, x_{n n}\right] / I$ мы будем обозначать через $\mathbb{Z}[G(\pi, \Sigma)]$.

ОпредЕлЕниЕ 2. Алгебраическая подгруппа в $G l_{n}(R)$,

$$
G(\pi, \Sigma, R)=\operatorname{Hom}_{\mathbb{Z}}(\mathbb{Z}[G(\pi, \Sigma)], R),
$$

называется группой Шевалле, соответствующей представлению $\pi$, системе $\Sigma$ и кольцу $R$.

Другими словами, группа $G(\pi, \Sigma, R)$ представляет соответствующую аффинную групповую схему Шевалле-Демазура над кольцом $R$.

Из сделанного определения непосредственным образом следует включение

$$
E(\pi, \Sigma, R) \subseteq G(\pi, L, R) .
$$

При этом, если $\Sigma$ является неразложимой системой ранга выше единицы, элементарная группа Шевалле нормальна в группе $G(\pi, \Sigma, R)($ см. [6]).

Для произвольного идеала $I$ кольца $R$ определены редукционный эпиморфизм $\Psi_{I}$ группы $G(\pi, \Sigma, R)$ и его ограничение на элементарную группу $\bar{\Psi}_{I}$, где

$$
\Psi_{I}: G(\pi, \Sigma, R) \rightarrow G(\pi, \Sigma, R / I), \quad \bar{\Psi}_{I}: E(\pi, \Sigma, R) \rightarrow E(\pi, \Sigma, R / I) .
$$

С гомоморфизмами $\Psi_{I}, \bar{\Psi}_{I}$ связаны центры по идеалу $I$ в группе Шевалле и в ее элементарной подгруппе:

$$
C_{I}(\pi, \Sigma, R)=\Psi_{I}^{-1}(C(G(\pi, \Sigma, R / I))), \quad \bar{C}_{I}(\pi, \Sigma, R)=\bar{\Psi}_{I}^{-1}(C(E(\pi, \Sigma, R / I))) .
$$


ОпредЕлениЕ 3 . Нормальная подгруппа $P$ группы $A$ называется первичной, если для произвольных $A_{1}, A_{2}, P \subseteq A_{i} \triangleleft A$, из условия $\left[A_{1}, A_{2}\right] \subseteq P$ следует, что для некоторого $i A_{i} \subseteq P$. Пересечение всех первичных нормальных подгрупп группы $A, b(A)$, называется первичным радикалом группы $A$. Группа $b(A)$ представляет собой множество всех строго энгелевых элементов $A$, т.е.

$$
b(A)=\left\{a \in A \mid \forall\left\{b_{i}\right\}_{i=0}^{\infty} \subseteq A, a_{0}=a, a_{i+1}=\left[\left[b_{i}, a_{i}\right], a_{i}\right], \exists j \geqslant 0, a_{j}=1\right\},
$$

где $[a, b]=a b a^{-1} b^{-1}$.

Первичный радикал группы $A$ тривиален в том и только том случае, когда $A$ не содержит нетривиальных нормальных абелевых (разрешимых) подгрупп.

ОПредЕЛЕниЕ 4. Будем говорить, что группа $A$ обладает разрешимым радикалом, если в $A$ определена наибольшая нормальная разрешимая подгруппа $\operatorname{Rad}(A)$.

Отметим, что данное определение корректно, поскольку наибольшая нормальная разрешимая подгруппа единственна.

В случае группы Шевалле над коммутативным кольцом связь между введенными радикалами групп и первичным радикалом кольца установлена в рамках следующей теоремы.

Теорема. Пусть $\Sigma$ - неразложимая система ранга выше единицы. Тогда для произвольного коммутативного кольца $R$

$$
b(G(\pi, \Sigma, R))=C_{B(R)}(\pi, \Sigma, R), \quad b(E(\pi, \Sigma, R))=\bar{C}_{B(R)}(\pi, \Sigma, R) .
$$

В случае $\Sigma=A_{1}$ указанные равенства выполняются при дополнительном условии: $2,3 \in G l(R)$.

СлЕДСТвиЕ. Пусть $R$ - коммутативное кольцо $c$ единицей, $\Sigma$ - неразложимая система неединичного ранга, отличная от $B_{2}$. Тогда разрешимый радикал группь $G(\pi, \Sigma, R)$ существует в том и только том случае, когда первичный радикал кольца $R$ нильпотентен. $B$ случае $\Sigma=B_{2}$ это утверждение имеет место при условии: $2 \in G l(R)$.

Пользуясь случаем, хочу выразить свою благодарность А.В. Михалёву, Н.А. Вавилову и Е. Б. Плоткину за внимание и ценные обсуждения.

\section{СПИСОК ЛИТЕРАТУРЫ}

[1] Vaserstein L. // Tôhoku Math. J. (2). 1986. V. 38. P. 219-230. [2] Abe E. // Contemp. Math. 1989. V. 83. Р. 1-17. [3] Стейнберг Р. Лекции о группах Шевалле. М.: Мир, 1975. [4] Chevalley C. Certains schemas des groupes semisimples // Sémin. Bourbaki. 1960/61. V. 13. Exp. 219. Paris. Secrétariat Mathématique. 1961. [5] Borel A. // Lecture Notes in Math. 1970. V. 131. P. 1-55. [6] Taddei G. // Contemp. Math. 1986. V. 55. P. 693-710. 